\title{
Continuing evolution of aerodynamic concept development using collaborative numerical and experimental evaluations
}

\author{
R M Cummings* and S A Morton \\ Department of Aeronautics, United States Air Force Academy, Colorado Springs, Colorado, USA
}

The manuscript was received on 6 December 2005 and was accepted after revision for publication on 24 February 2006.

DOI: 10.1243/09544100JAERO71

\begin{abstract}
Traditionally, computational predictions and experimental evaluations of aerodynamic concepts have been conducted separately, with little collaboration other than post priori comparisons of results. This has led to distrust and even antagonism between the computational and the experimental communities. These difficulties probably began when early computational fluid dynamic practitioners boasted that wind tunnels would become secondary in aerodynamic concept development within a few short years, a prediction that has not come true. On the contrary, it is believed that a great deal of synergy can be cultivated when computational and experimental evaluations are conducted in an integrative fashion. A variety of projects where this has been done will be reviewed, including a pitching Unmanned Combat Air Vehicle, a delta wing with periodic suction and blowing for aerodynamic control, a missile with drag brakes that caused excessive unsteady flow, a C-130 aircraft configured for airdrop, and closed-loop flow control. Further evolution of the numerical/experimental collaboration will be discussed showing results from the flow control research where the dividing line between numerical predictions and experimental evaluations is becoming blurred. Suggestions for future directions in collaboration will also be made.
\end{abstract}

Keywords: computational fluid dynamics, experimental aerodynamics, unsteady aerodynamics

\section{INTRODUCTION}

In 2003, a conference was held at the University of Glasgow to honour the retirement of Professor Bryan Richards, who had spent a considerable portion of his career encouraging collaboration between computational and experimental research, especially the importance of seeing computational fluid dynamics (CFD) as an applied (and therefore quite useful) tool. One of the foundational principles of the meeting was eloquently stated in this excerpt from the conference:

'It could be argued that the process of aerodynamic investigation would be significantly enhanced if the integration of CFD and experiments was much

\footnotetext{
${ }^{*}$ Corresponding author: 2354 Fairchild Drive, Department of Aeronautics, United States Air Force Academy, Suite 6H27, Colorado Springs, Colorado 80840,USA.email: russ.cummings@ usafa.edu
}

stronger. In particular, the design and reliability of experiments could be significantly enhanced by CFD, the scope of experimental measurements extended through CFD and the credibility of the simulation results enhanced by the availability of suitable measurements from experiments. This sort of closer integration is however rare'.

Quote from 1st Integrating CFD and Experiments in Aerodynamics Conference, Glasgow Scotland, September 2003

The purpose of this paper is to investigate the status of this type of aerodynamic collaboration and to see whether improvements in aerodynamic concepts might result from improvements in how the research is performed. Unfortunately, this situation (of 'rare' collaboration) arises due to various historical occurrences in the past 30 years.

At a major conference at the NASA Langley Research Center in 1975, Chapman [1] drew on the rapid advances in computer capability to present a chart that could be used to project that 
computational aerodynamics would be fully developed in 1985, as shown in Fig. 1. Chapman's statement was based on Moore's law (the doubling of computer processor speed every 18 months) and an estimate of the grid size necessary to model a three-dimensional wake for a full aircraft using the Reynolds-averaged Navier-Stokes (RANS) methods. Although Chapman's estimate was probably correct (although limited by the usefulness of such computations), the impact of his comments was far more important than the truth of his statement. Chapman's statement, and those made by many others, created a rift between CFD and experimentalists that continues to the present day. Chapman made a statement that, in particular, upset a great many people $[\mathbf{1}]:$ ' . . If history is a guide, the wind tunnels can be expected to play a secondary role to the computers in aerodynamics ....

Experimentalists, naturally, reacted by buttressing their fortress and attacking CFD (an attack that continues to the present). A long list of complaints about CFD were quickly generated and used often to challenge the appropriateness and accuracy of various CFD predictions. While many of these 'complaints' were valid, the natural impact was that CFD researchers went off and found their own way, working with experimentalists as little as possible. This led to a great deal of derision and animosity between the two research communities, resulting in, among other things, jokes made at the expense of the other camp. Here is a joke by the CFD researchers at the NASA Ames in the mid 1970s that reflects the attitude of the time. Question: 'What do you use wind tunnels for'? Answer: 'They are places with lots of space, where you store your computer output'.

Very few people made any efforts to form links between the experimental and the computational world, and fewer people actually collaborated. CFD researchers tended to see experimentalists only as suppliers of data for code validation, and

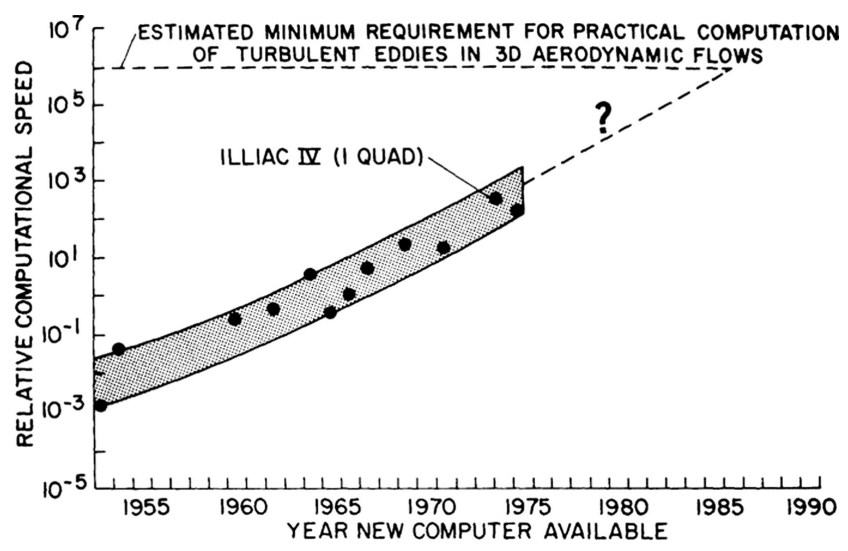

Fig. 1 Estimate of computer requirements for practical CFD predictions [1] experimentalists saw them as upstarts who siphoned away valuable resources and support. Unfortunately, a great opportunity for advancing the aerodynamic knowledge was lost because of the rift that was formed between the two communities. Few people had the foresight to realize that both experiments and computations have advantages and disadvantages and that each community could offer insight into aerodynamic concepts that would be strengthened by the presence of the other group's insight. A possible synergistic relationship between the two communities was replaced by stone throwing and isolation. The authors' strong opinion is that the collaborative research between the two communities has the ability to offer impetus to the development of aerodynamic concepts and designs. The remainder of this paper discusses how the synergy could take place and gives several examples where integrated research has paid large dividends.

\section{ADVANTAGES AND DISADVANTAGES OF EXPERIMENTS AND COMPUTATIONS}

It is imperative that people conducting joint computational/experimental research should be well versed in the advantages and disadvantages of both the computational approach and the experimental procedure (including error analysis). Without this knowledge at hand, collaboration is more difficult and a great deal of time can be wasted trying to resolve discrepancies in results. Some of the advantages and disadvantages of both experiments and computations are briefly discussed.

\subsection{Experiments}

All experimental methods have various advantages and disadvantages; however, there are a number of issues that come to mind immediately. Certainly, any list has its own deficiencies, so the authors invite the reader to add their own ideas to these lists. Some of the strong advantages of wind tunnel testing include:

(a) well-known and understood capabilities;

(b) usually easy to set and verify free stream conditions;

(c) forces and moments are relatively easy to obtain;

(d) flow-field properties are readily available (from probes, hot wire, laser dopler velocimetry (LDV), particle image velocimetry (PIV), pressure sensitive paint (PSP), etc.).

Some of the disadvantages of wind tunnel testing include:

(a) many measurements are intrusive and modify the flow; 
(b) wall corrections are often required and difficult to make;

(c) support system corrections are often required and difficult to make;

(d) blockage issues must be addressed;

(e) model fidelity is often a challenge;

(f) matching flight conditions can be difficult (Reynolds number, transition, etc.);

(g) transonic flow is especially troublesome because of nearly normal shocks.

\subsection{Computations}

Perhaps, the biggest disadvantage to CFD predictions is the over-optimism of the earliest users, as described in Introduction. In fact, CFD's non-acceptance by many people led to a common lament among CFD practitioners: "no one believes CFD results except the person who ran the code and everyone believes wind tunnel results except the person who conducted the test'. There is certainly a great deal of truth to this, but in reality, there are a number of advantages and some important disadvantages to CFD. Some of the advantages of CFD include:

(a) complete flow-field prediction (all properties are predicted throughout the flow);

(b) matching flight conditions is fairly straightforward;

(c) Non-intrusive flow-field 'measurements' can be made;

(d) steady or time-accurate results are possible;

(e) flow visualization is relatively easy.

Some of the disadvantages of CFD include:

(a) turbulence modelling;

(b) transition prediction;

(c) numerical dissipation;

(d) numerical error;

(e) 'black box' syndrome (garbage-in-garbage-out is still a common CFD problem).

Without understanding the strengths and weaknesses of both approaches, researchers are left to 'grope' in the dark at gaining understanding into various aerodynamic phenomenon - using both approaches is often enlightening and beneficial to understanding. Several examples of collaboration will be shown, which detail how experiments and CFD can be used together and how an evolution is taking place that utilizes both approaches to their fullest capability in aerodynamic design. These examples are approximately presented in the chronological order to show how collaboration is evolving and improving.

\section{DELTA WING WITH PERIODIC SUCTION AND BLOWING FOR FLOW CONTROL}

The first example presented here of a situation where close collaboration between experimentalists and computationalists paid dividends is for a delta wing study conducted at the US Air Force (USAF) Academy. The purpose was to determine the feasibility of using periodic suction and blowing (PSB) along the leading edge of the wing $[2,3]$. The $70^{\circ}$ delta wing configuration was tested in the USAF Academy water tunnel at $\alpha=35^{\circ}$ and $R e_{\mathrm{c}}=40700$ (Fig. 2). The wing has a chord length of $298 \mathrm{~mm}$, is hollow, and has a $1.5 \mathrm{~mm}$ slot along the entire leading edge, through which the suction and blowing are actuated normal to the wing leading edge. Two-dimensional PIV measurements were taken of the flow over the upper surface of the delta wing, but no force and moment data were taken.

To perturb the shear layer originating at the leading edge of the delta wing, a semi-spherical rubber cap was used as an oscillatory blowing and suction flow actuator. It was moved back and forth by a connecting rod, eccentrically mounted on a disc that was driven by a $560 \mathrm{~W}$ DC motor. The water displacement produced by the moving cap was channelled through a tube $2 \mathrm{~cm}$ in diameter to the hollow wing and to the length of the slot in its leading edge. With this setup, as with any oscillatory flow control method, fluid is drawn into the actuator over one half of the sinusoidal cycle and ejected over the other half $\left(V=V_{\mathrm{o}} \sin \omega t\right)$. The phase during the forcing cycle is determined by the position of the rotating disc flywheel, which features an adjustable optical pickup to synchronize the data acquisition with a particular phase of the forcing cycle. A forcing cycle starts at $0^{\circ}$ with the blowing phase which extends to $180^{\circ}$. The suction portion between $180^{\circ}$ and $360^{\circ}$ completes the cycle.

One of the problems encountered during the experimental phase of the investigation was that it

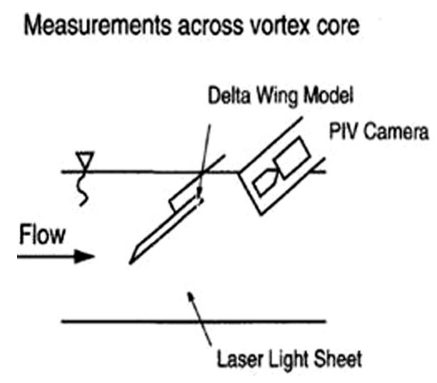

Side View of Test Section
Measurements along vortex core

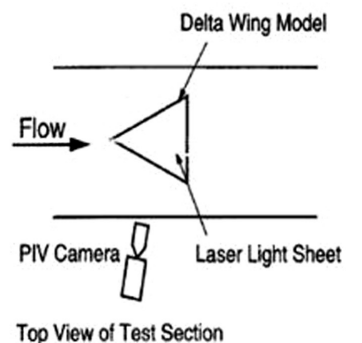

Too View of Test Section
Fig. 2 PIV measurements of the delta wing in wind tunnel $[2,3]$ 
did not appear that the suction phase was equally as effective as the blowing phase of the periodic cycle. Although this observation was important to the experiment, no direct reasons for the apparent anomaly were known, leaving the experimentalists to wonder whether their apparatus was operating correctly or whether there was some fluid dynamic interaction at work. Another difficulty realized by the researchers was that although they knew the impact of the suction and blowing on the flow field, they did not know the impact on the aerodynamic forces of the delta wing.

The CFD solutions for the delta wing (as well as all case studies in this paper) were performed using the unstructured flow solver Cobalt. Cobalt solves the Navier-Stokes equations, including an improved spatial operator and temporal integration. The code has been validated on a number of problems, including those that use the Spalart-Allmaras turbulence model, which forms the core of the Detached-Eddy Simulation (DES) hybrid turbulence model [4]. Tomaro et al. [5] converted Cobalt from explicit to implicit, enabling Courant-Friedrichs-Lewy (CFL) numbers as high as one million. Grismer et al. [6] then parallelized the code, yielding a linear speedup on as many as 1024 processors. Forsythe et al. [7] provided a comprehensive testing and validation of the RANS models, including the Spalart-Allmaras, Wilcox $k-\omega$, and Menter's turbulence models.

Results from the CFD simulation for the flow field around the delta wing show multiple frequencies in the normal force variation for the converged PSB case, as shown in Fig. 3 [2, 3]. These cases were run

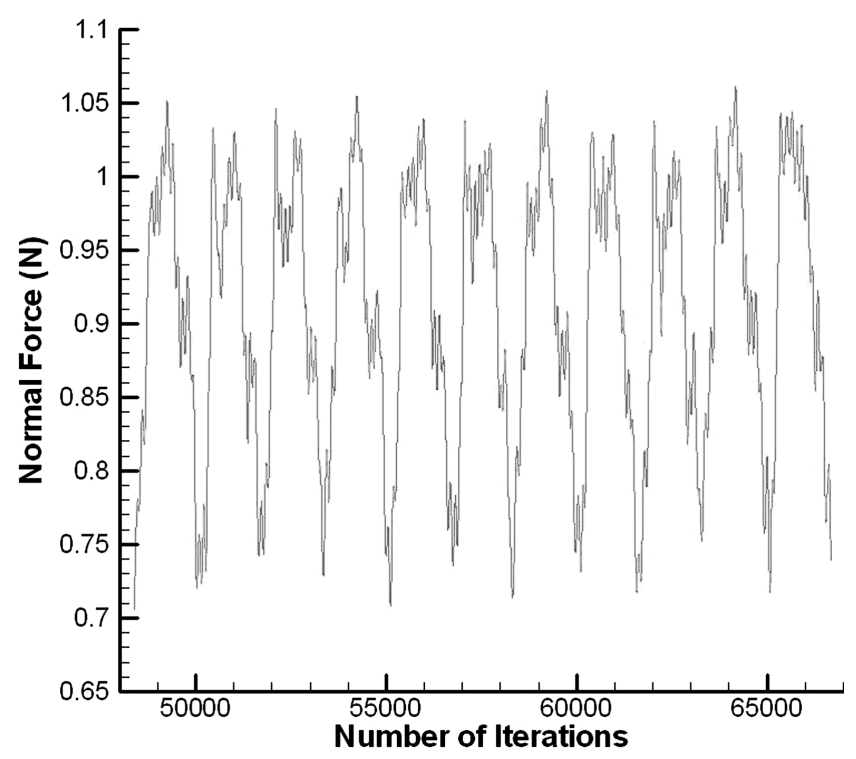

Fig. 3 Normal force variation for periodic suction and blowing, $\Delta t^{*}=0.006[2,3]$. (a) Blowing phase, $90^{\circ}$ and (b) suction phase, $270^{\circ}$ time accurate and the results show the normal force variation for 17000 iterations (over ten cycles of the suction and blowing). The suction and blowing frequency is obvious, but overlayed on that frequency is the shear-layer instability frequency, constantly oscillating around the lower frequency. Also note that the blowing portion of the suction/blowing cycle is more effective, as evidenced by the amount of time the normal force remains at the highest levels. When the suction cycle takes place, decreasing the normal force, the force spikes to a minimum value, but then quickly rises again as the suction phase ends. This explains why, during the experimental portion of this work, it appeared that the suction was incomplete (or possibly working incorrectly). Even the numerical simulation clearly shows that the suction phase is not as effective in altering the normal force acting on the delta wing. There is also a slight dwell as suction begins, which was also not explained by the PIV results.

Again, the CFD results were able to answer some of these questions because of the ability to interrogate the flow field at all locations and at all times. Figure 4 shows the velocity vectors in the vicinity of the delta wing leading edge at the 60 per cent chord location. During the blowing phase $\left(90^{\circ}\right)$, Fig. 4(a) shows that the fluid is able to expel directly into the surrounding flow and have a direct impact on the shear layer region. However, during the suction phase $\left(270^{\circ}\right)$, Fig. 4 (b) shows that the flow in the vicinity of the leading edge of the delta wing is not able to turn the sharp corner and be fully brought into the PSB channel. This explains the difference seen in Fig. 4 between suction and blowing and may also explain the dwell during the blowing phase, as the flow is attached and fully formed during this phase.

Although the CFD was able to answer these questions from the experiment, the CFD did not fully match all the experimental data, especially in the postbreakdown region of the delta wing primary vortices. The qualitative details of the flow field matched quite well, whereas specific measured properties (such as velocity components) did not match at all chordwise locations and at all times during the PSB cycle. This was in spite of the fact that an intensive study of the time steps used in the CFD simulation had been performed. It may well be that the massively separated flow-field downstream of breakdown would require an even finer grid to match the experimental results, something that has been shown quite dramatically in reference [8]. In hindsight, this type of study would have benefited greatly from conducting the numerical simulation and experimental evaluation in parallel, rather than in series, something that is learnt and put into effect in later research work. Specifically, a great deal of time would not have been wasted during the 

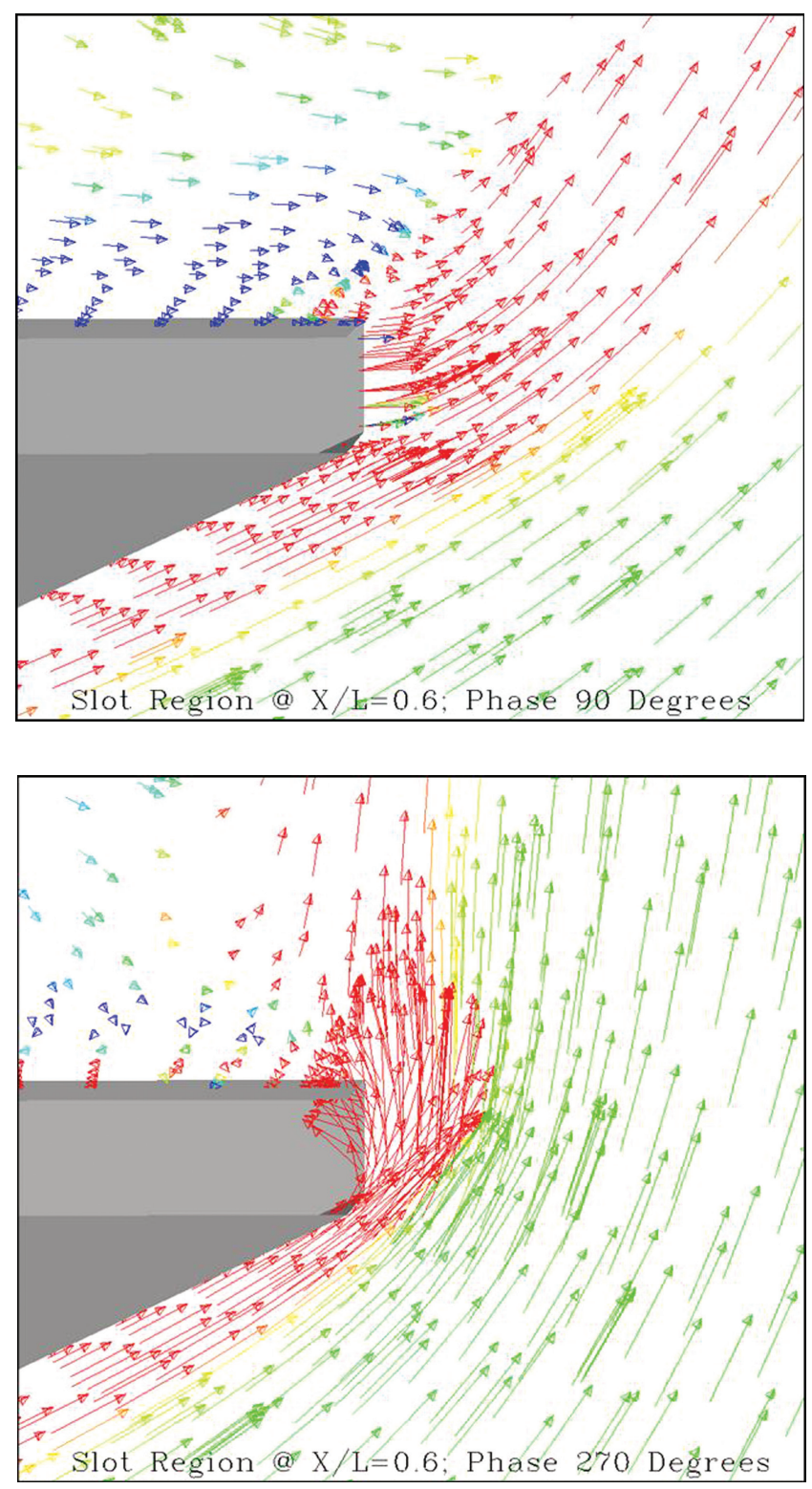

Fig. 4 Velocity vectors near delta wing leading edge showing difference between suction and blowing $[2,3]$

experiment in evaluating the PSB apparatus and whether it was working properly. This would have left more time to collect and analyse data. Also, as the forces acting on the delta wing ended up being an important piece of data, the experiment might have been expanded to include a force balance phase, which then would have provided a more comprehensive set of data for comparison with the CFD.

\section{PITCHING UNMANNED COMBAT AIR VEHICLE CONFIGURATION}

A full-scale model for the Boeing 1301 Unmanned Combat Air Vehicle (UCAV) configuration is shown in Fig. 5; the configuration has many similar features to the X-45A UCAV configuration. The 1301 configuration has a straight, $50^{\circ}$ sweep leading edge, an aspect ratio of 3.1, rounded leading edges, a topmounted engine inlet, and a blended wing/body planform. A 1:46.2 scale model of the configuration was tested in the USAF Academy $0.914 \mathrm{~m} \times 0.914 \mathrm{~m}$ open return low-speed wind tunnel [9]. The scaled model has a mean aerodynamic chord of $0.133 \mathrm{~m}$ and a reference area (wing planform area) of $302.1 \mathrm{~cm}^{2}$. The tunnel has $<0.05$ per cent free stream turbulence levels at all speeds. The test was conducted at a free stream velocity of $20 \mathrm{~m} / \mathrm{s}$, which corresponds to a chord-based Reynolds number of $1.42 \times 10^{5}$. The model was sting-mounted from the rear, and forces and moments were measured with a six-component force balance. Both static testing and dynamic testing were done; forces during the dynamic runs were obtained by subtracting the force history with the tunnel off from the dynamic data. The dynamic pitching was done with a shifted cosine oscillation, starting at a certain angle of attack and pitching up to twice the peak amplitude of the cosine wave, then back to the original angle of attack

$$
\alpha(t)=\alpha^{\circ}+m^{\circ}-m^{\circ} \cos (\omega t)
$$

where $\alpha^{\circ}$ and $m$ were varied to obtain results for $0^{\circ} \leqslant \alpha \leqslant 45^{\circ}$ in three pitching cycles. This pitching function was used because it produces a motion without any discontinuities in acceleration or velocity at the beginning and end of the motion, thus being easier to implement in an experiment or a CFD code.

One of the limitations and difficulties encountered during the experiment was that the experiment only was able to measure forces. This is a common problem during wind tunnel tests, where tests are either of the force and moment variety or of the flow-field property variety, but rarely does an experiment include both sets of measurements. Because of this, researchers are usually unsure of the fluid dynamic causes of various results, being left to make educated guesses about unusual or unexpected results. For example, in the case of the UCAV wind tunnel tests, the vehicle lift coefficient showed linear lift characteristics up to $\sim 10^{\circ}-12^{\circ}$ angle of attack, as shown in Fig. 6. Wing stall was evident at $\sim 20^{\circ}$ angle of attack, with the lift being re-established up to $32^{\circ}$, after which an abrupt loss of lift takes place. What is the cause of the poor lift characteristics? Are the results a direct effect of leading-edge vortices and vortex breakdown? The experimentalist is left to hypothesize and wonder, but the numerical researcher can add insight into the problem. 

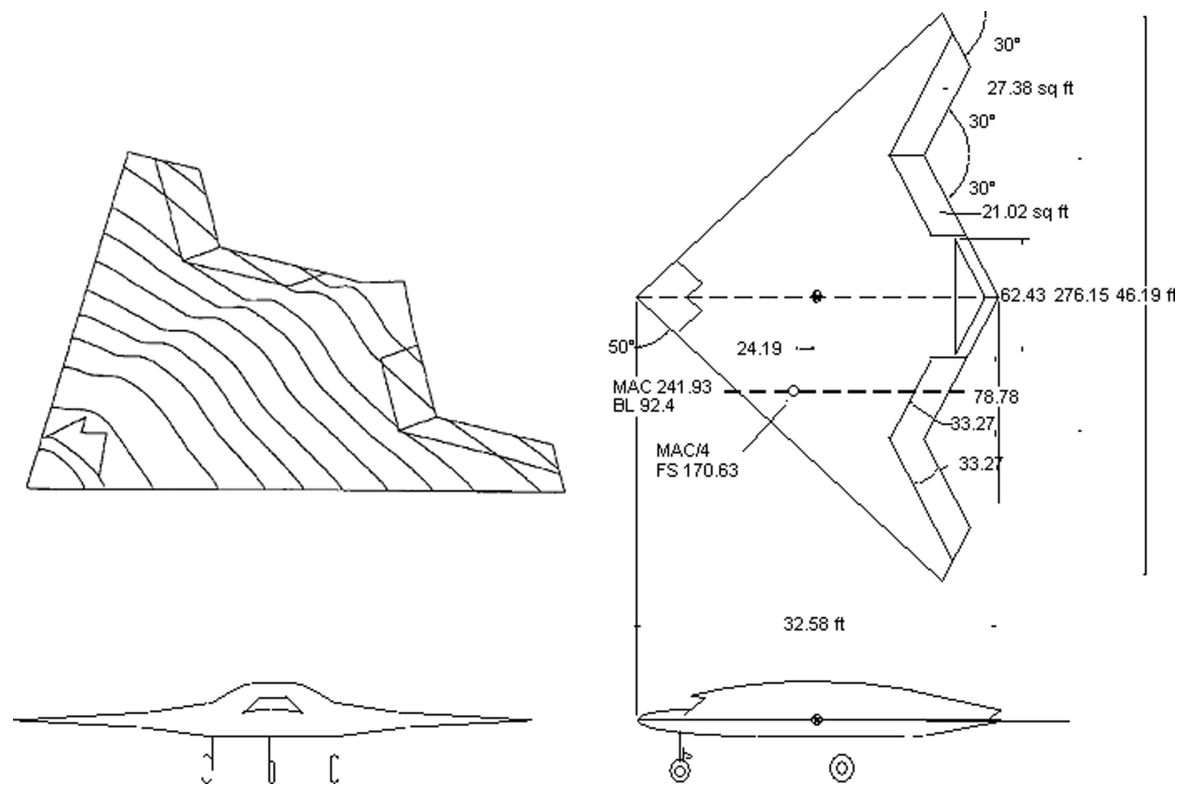

Fig. 5 Boeing 1301 UCAV configuration [9]

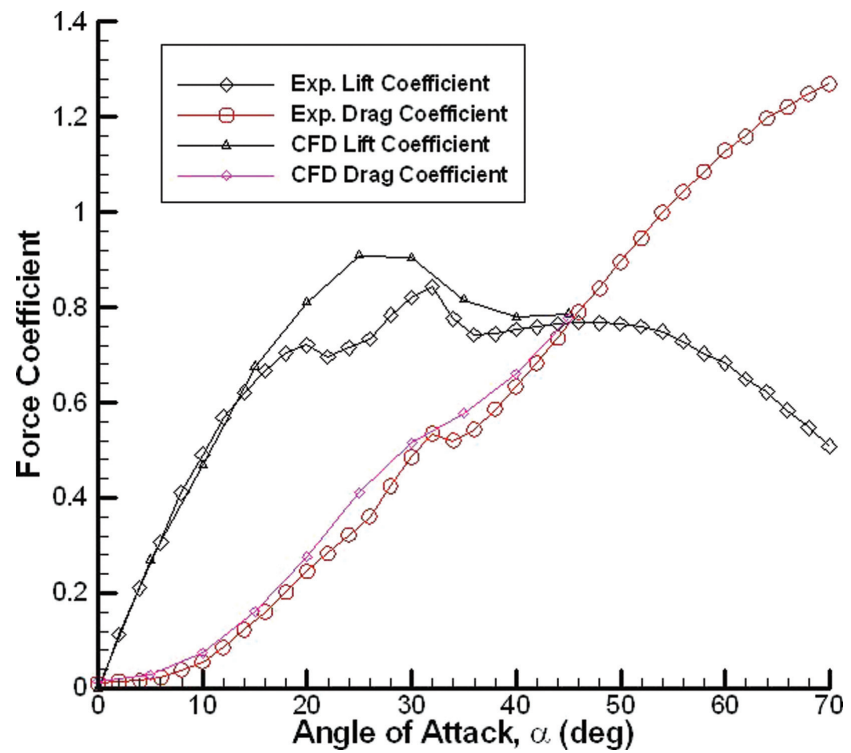

Fig. 6 Numerical (time accurate) and experimental (static) force coefficient comparison [9]

Figure 6 shows the results of the CFD predictions. Perhaps, the most important result of the CFD simulation was the realization of just how unsteady the flow fields in the poststall region were. Timeaccurate results matched the experiment fairly well, with fairly good modelling of the flow field, including drag, up to $\alpha=45^{\circ}$. However, there was a difference in lift from $\alpha=20^{\circ}$ to $30^{\circ}$, which could have been caused by the presence of the sting, surface roughness, transition, or a host of other phenomenon. Additionally, the impact of transition to turbulence is difficult to determine in this case. Although the chord-based Reynolds number was $1.42 \times 10^{5}$ and the CFD simulation was conducted using the laminar Navier-Stokes equations, there was no way to know if and when the flow field was turbulent during the experiment. In fact, it was certain that the presence of turbulent flow was highly unsteady and extremely difficult to predict. This type of difficulty further strengthens the argument that the study should be conducted with both experiments and CFD simulations in order to gain the greatest possible understanding about the flow.

Figure 7 shows representative numerical simulations of the configuration at $\alpha=5^{\circ}, 10^{\circ}, 15^{\circ}$, and $20^{\circ}$, with the flow field being visualized with streamlines and the surface coloured with pressure. The leading-edge vortices are clearly visible closely following the $50^{\circ}$ sweep, until approximately $x / l=0.40$ when vortex breakdown is evident. Low surface pressures are visible beneath the vortex prior to breakdown; these low pressures account for the lift on the configuration at $\alpha=20^{\circ}$. After breakdown, the vortex wake quickly moves up and behind the leading edge, leading to higher pressures on the upper surface of the wing. The vortices are very wide compared with their height, most likely due to the rounded leading edges of the wing, and there may even be two vortices present. Secondary vortices are also visible beneath the primary vortices. The primary vortex is seen splitting into two flow structures after the breakdown location.

These numerical simulations help to answer some of the questions raised by the wind tunnel tests. The rounded leading edges and mid-range leading-edge sweep yield weak leading-edge vortices that do not produce very much lift. The vortices are only just beginning to form (and are very weak) when 


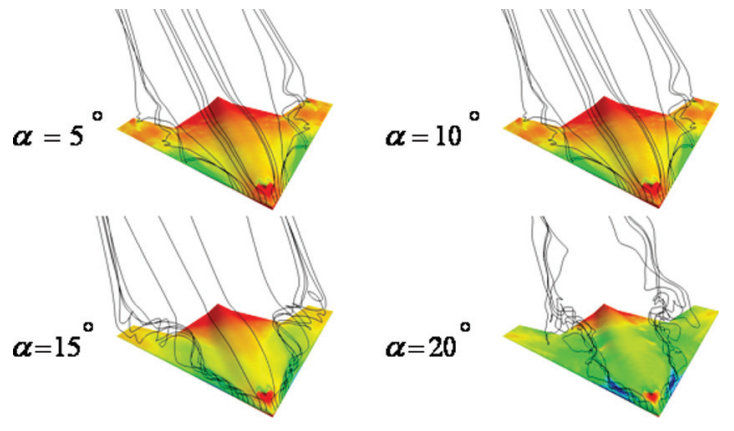

Fig. 7 Numerical flow-field predictions for various angles of attack (surface coloured by pressure) [9]

breakdown takes place and reduces lifting benefit of the vortices. These are common characteristics of 'lambda' type wings, but seeing the CFD simulation helps to place understanding into the wind tunnel test data.

\section{ARGUS MISSILE CONFIGURATION}

The Advanced Remote Ground Unattended Sensor (ARGUS) missile configuration was tested in the subsonic wind tunnel at the USAF Academy (as shown in Fig. 8), where a companion CFD study was also completed $[\mathbf{1 0}, \mathbf{1 1}]$. A 61.5 per cent model tested in was tested in the USAF Academy's subsonic wind tunnel for $M_{\infty}=0.2-0.5$, at angles of attack ranging from $\alpha=-5^{\circ}$ to $20^{\circ}$, and for a length-based Reynolds number of $R e_{\mathrm{L}}=3.5 \times 10^{6}$ to $8.7 \times 10^{6}$. The missile has drag brakes that are used to control velocity, which is critical to the overall mission of the vehicle.

The configuration had a number of unusual aerodynamic features, including a production of negative lift at positive angles of attack and a substantial coning motion during flight. Although the wind tunnel test could give results that verified these behaviours, the test could not explain why the missile behaved as it did. Figure 9 shows the CFD predictions for the configuration at a free stream Mach number of 0.5 . Although the normal force coefficient variation with angle of attack looks reasonable, the CFD also predicted that the configuration produced negative lift as a function of angle of attack up to about $\alpha=15^{\circ}$. Again, the wind tunnel results did not shed any light on why this happened.

One of the unique features of CFD is the ability to account for forces on a variety of surfaces in the flow. For example, it was possible to account for forces on the missile body as well as the drag brakes, which might shed light on the cause of the negative lift coefficients. Figure 10 shows the total lift coefficient, as well as the components due to the body and the fins. It is fairly obvious that the body is

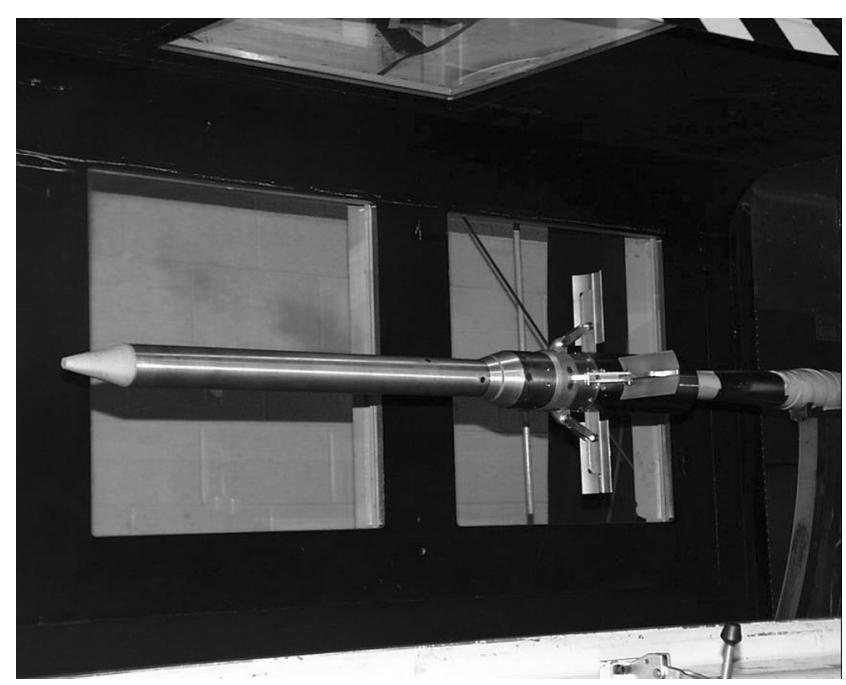

Fig. 8 The ARGUS configuration in the USAF Academy subsonic wind tunnel

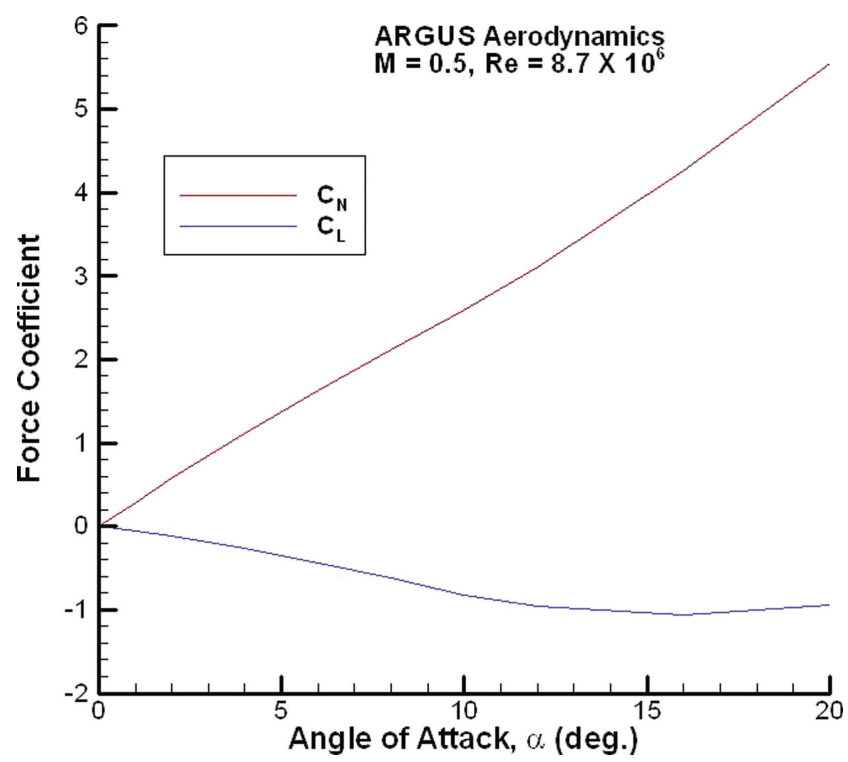

Fig. 9 The ARGUS normal force and lift coefficients as a function of angle of attack $[10,11]$

producing lift normally and that the negative lift is coming from the fins. What is not very obvious is why the fins are producing negative lift. However, an evaluation of the flow field can quickly answer this question.

Flow visualization in Fig. 11 begins to shine light on the situation, as the curved drag brake extension arm is seen creating a region of low pressure on the upper surface of the fin at $\alpha=20^{\circ}$. The vorticity contours in the vicinity of the drag brake show flow separation over the support arm, which extends over most of the upper half of the brake. Therefore, the lower surface of the brake has attached flow, but the curved nature of the brake creates a negative lift coefficient, whereas the upper surface of the 


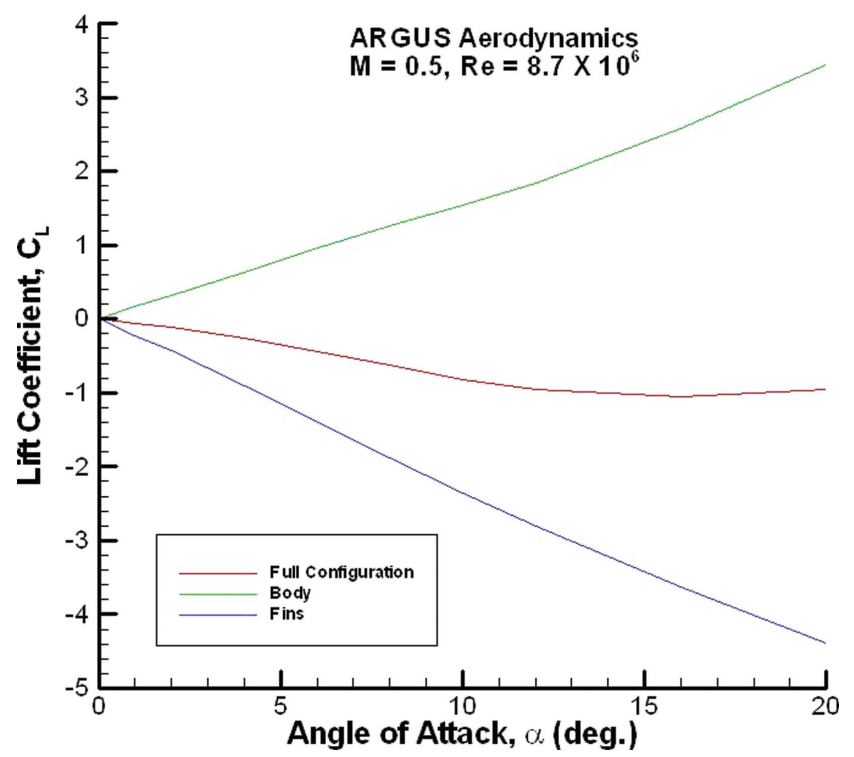

Fig. 10 The ARGUS lift coefficient component breakdown $[\mathbf{1 0}, \mathbf{1 1}]$

brake has separated flow and does not counter the force or moment created on the lower surface.

One of the unforeseen problems with the drag brakes was the unsteady forces and moments on the configuration, leading to yaw-roll coupling and coning motion during flight. To alleviate this problem, perforations were added to the drag brakes, but a basic understanding of the fluid dynamic reasons for the flow was not known from the wind tunnel test. Figure 12(a) shows the time-accurate DES of the baseline drag brakes, and when animated, it becomes clear that the blunt drag brakes are creating vortex rings, which shed from each brake at different times - a classic vortex-shedding flow field. Figure 12(b) shows the results for perforated drag brakes (the same isosurface levels were used for both figures) where now vortex shedding is evident, even though there are still low levels of flow unsteadiness. The perforations solved the vortexshedding problem and alleviated the coning motion of the configuration.

This was a case where CFD and wind tunnel investigations were done in parallel, and the resulting improvement in the ARGUS configuration was largely due to the collaboration. Researchers were obtaining and sharing results with one another throughout the program, which resulted in important design decisions being made with higher levels of confidence. At no time during this project, one set of researchers (experimental or numerical) left wondering what their results meant - they had nearly instant access to the other researcher's information so that problems could be analysed and solutions could be obtained.
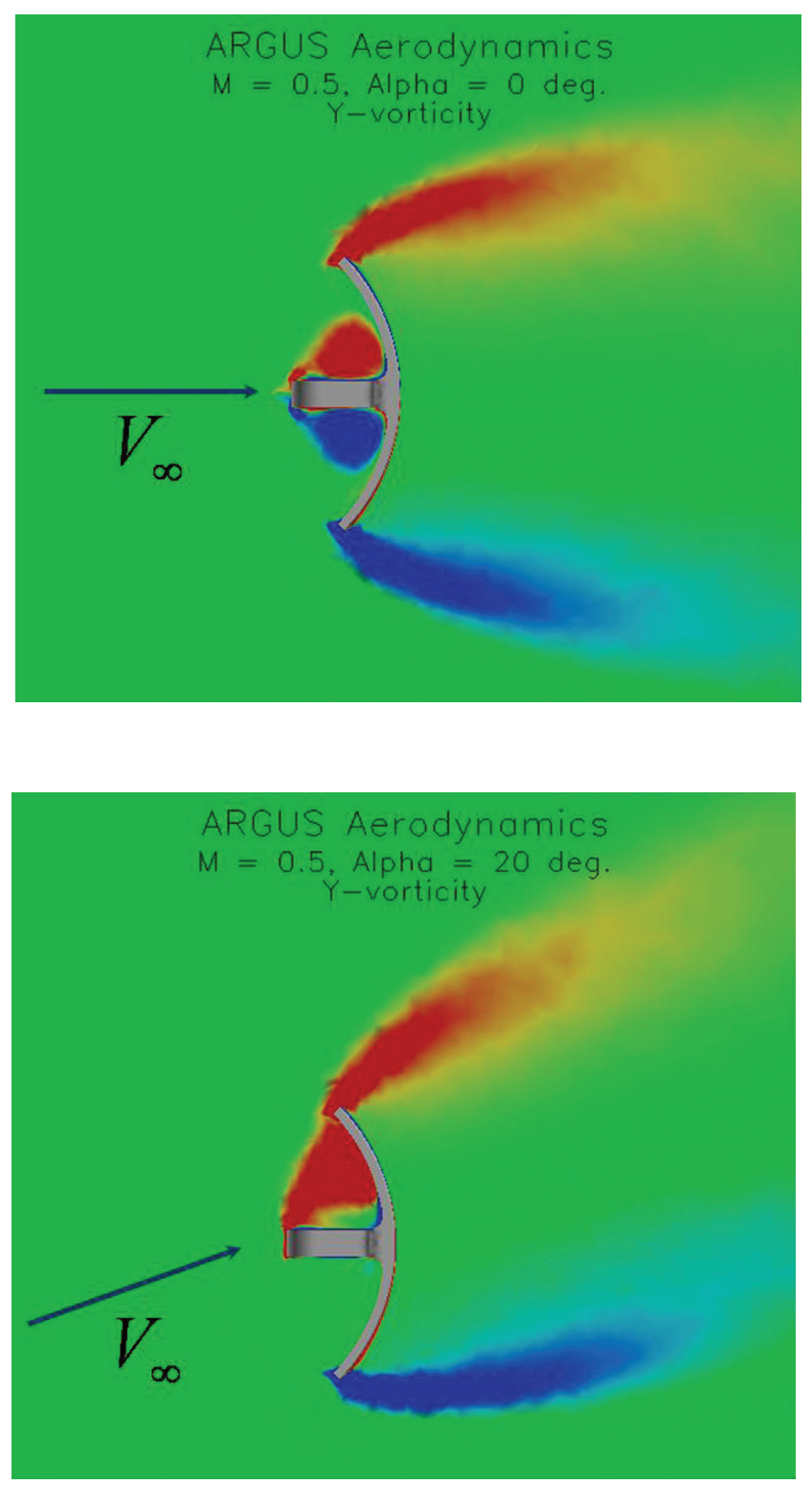

Fig. 11 Flow field in the vicinity of the ARGUS fins coloured by y-vorticity $[\mathbf{1 0}, \mathbf{1 1}]$. (a) Baseline brakes and (b) brakes with holes

\section{C-130 AIRDROP CONFIGURATION}

This is also a case where CFD and experiments were being conducted in a collaborative fashion. A wind tunnel model of a C-130 was being tested at various wind tunnels in different countries, with different types of flow-field tests being conducted at each tunnel (force and moments, surface flow visualization, etc.). As the wind tunnel tests preceded, the results were in disagreement with the CFD simulations being performed, leading to a great deal of hand wringing and consternation. Finally, after a lot of hard work, it was discovered that the wind tunnel model had been degrading in shape as various 

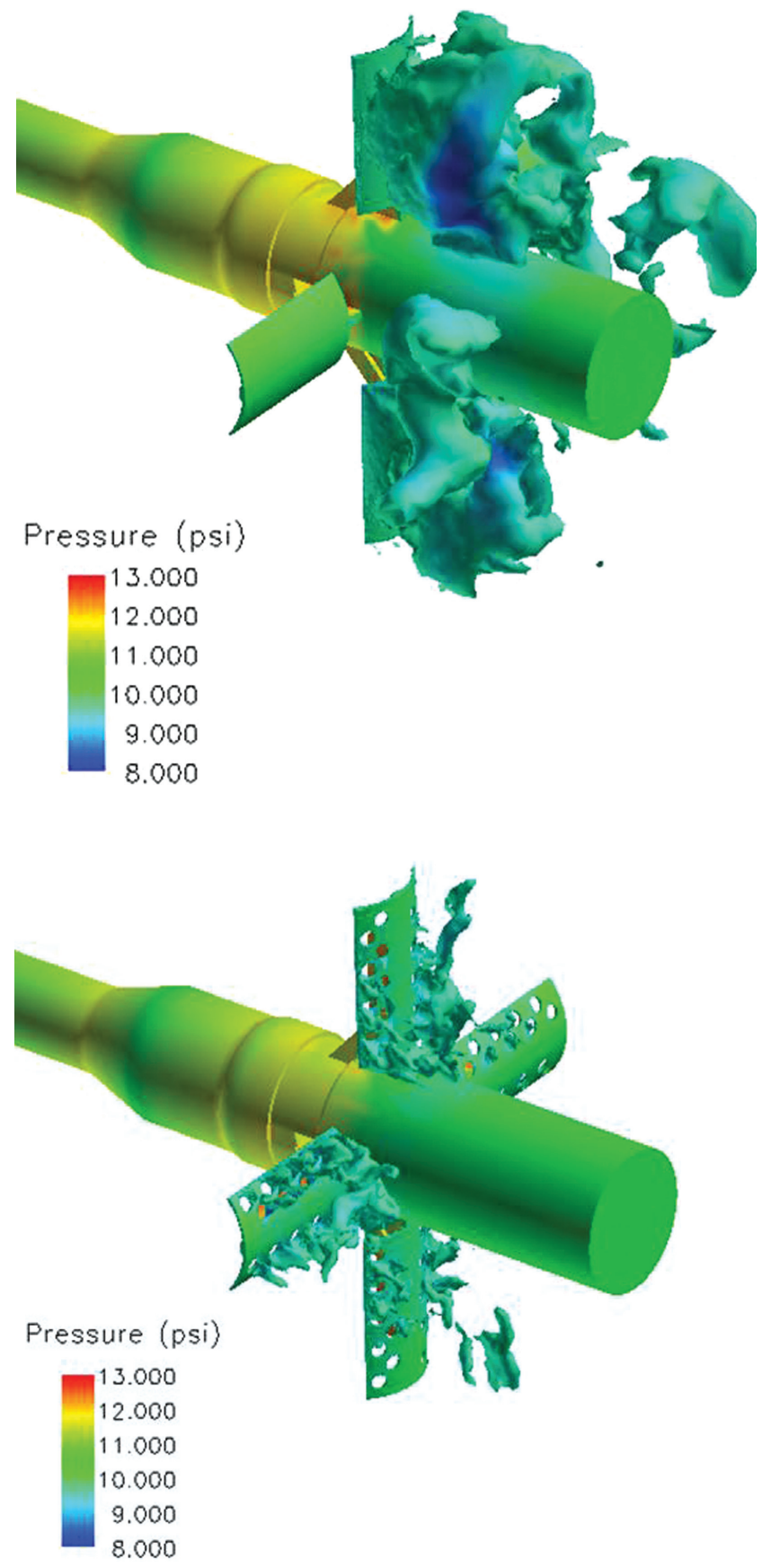

Fig. 12 DES simulation of flow field in the vicinity of the ARGUS fins $[\mathbf{1 0}, \mathbf{1 1}]$

tests were being performed (Fig. 13), leading to more and more configuration mismatch between the model and the original CAD description which had been used to create the CFD grids.

This led to a study of the model material's chemical compatibility with products to be used in the wind tunnel tests, including (possibly):

(a) fluorescent viscous wall coatings;

(b) acetone;

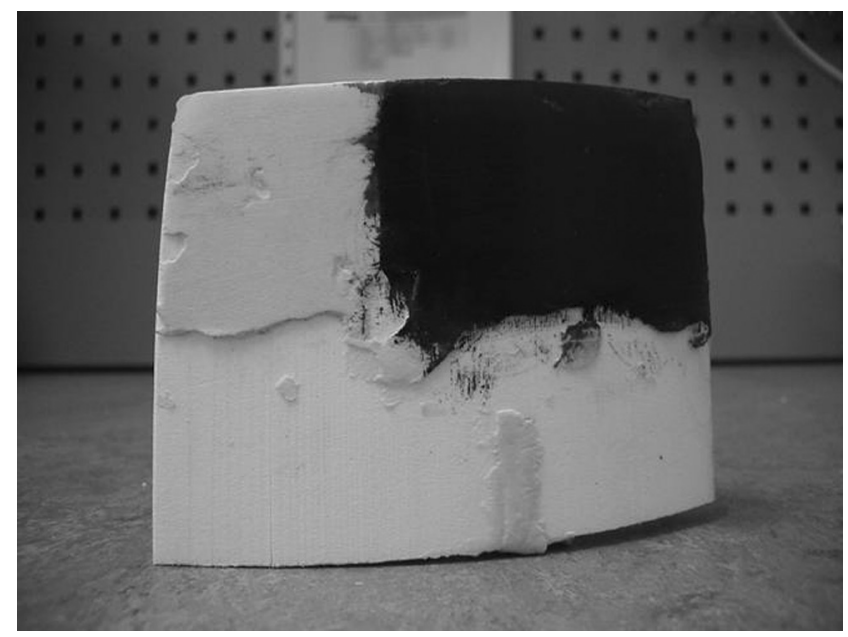

Fig. 13 The C-130 wind tunnel model part showing the decomposition of surface shape

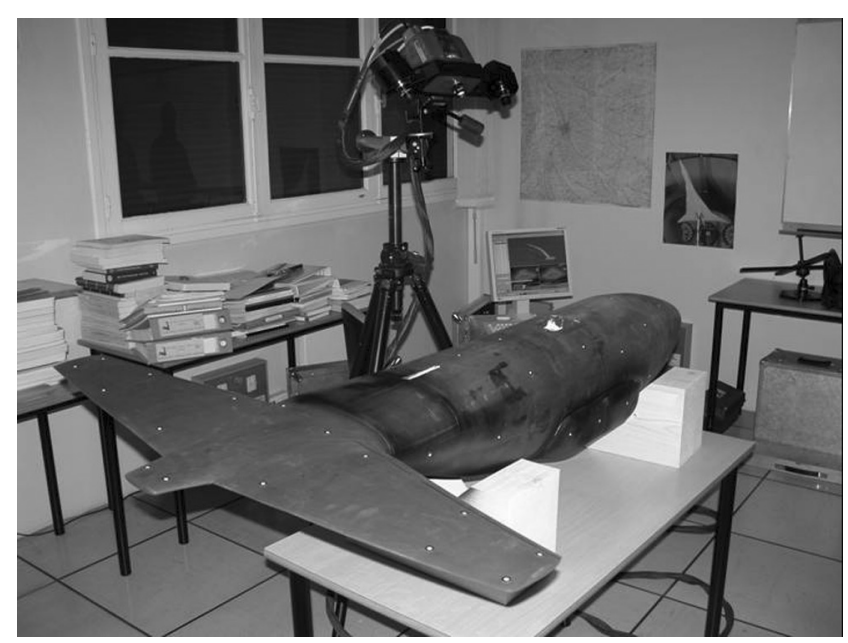

Fig. 14 Three-dimensional optical digitizing of the C-130 wind tunnel model

(c) black paint;

(d) fog generating liquid;

(e) filling of holes and gaps with putty

A three-dimensional optical digitizing of the wind tunnel model was performed at the ENSICA (achieved by GOM Company, as shown in Fig. 14) in Toulouse, and comparisons were made with the original CAD geometry. Figure 15 shows the differences between the original CAD geometry and the actual model being tested in the ENSICA wind tunnels, with large variations evident at various locations around the fuselage and horizontal tails. In fact, the tips of the horizontal tails appear to be bent as much as $2 \mathrm{~mm}$ away from the original CAD shape. This shows another example of how CFD and experiments can be used together to ensure accuracy of results in aerodynamic evaluation. 

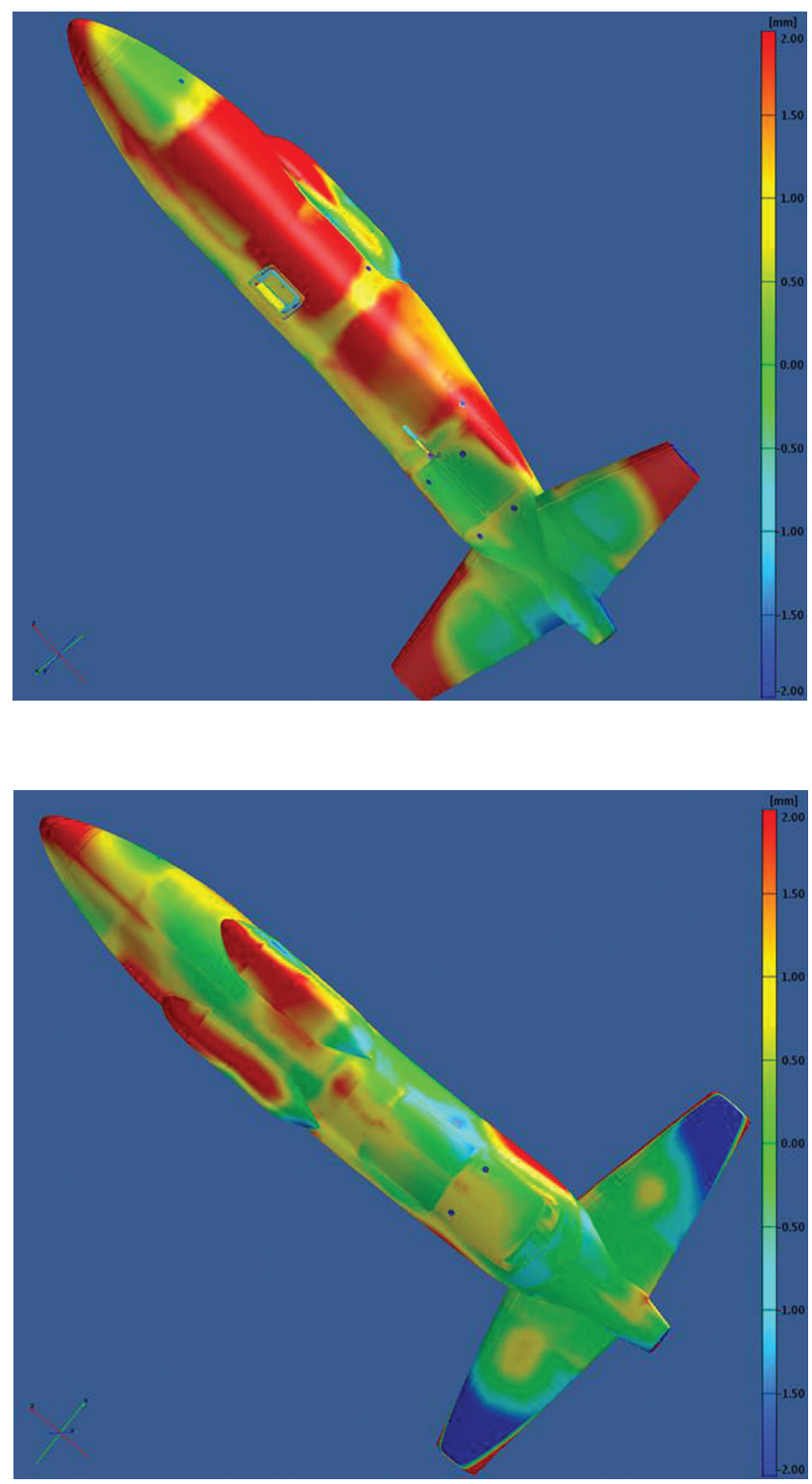

Fig. 15 Differences between the C-130 wind tunnel model and original CAD definition: coloured scale: -2 to $+2 \mathrm{~mm}$

\section{CLOSED-LOOP FLOW CONTROL}

The final example of the evolution of collaboration between experiments and numerical simulations is research being conducted in closed-loop flow control. A novel combination of numerical and experimental evaluations is being conducted to show the effect of feedback flow control on the wake of a circular cylinder at a Reynolds number of 120, as shown in Fig. 16 [12-14]. An initial two-dimensional numerical simulation of the laminar flow was investigated using proper orthogonal decomposition (POD) by placing sensors at various locations downstream of the cylinder, as shown in Fig. 17. The flow was also

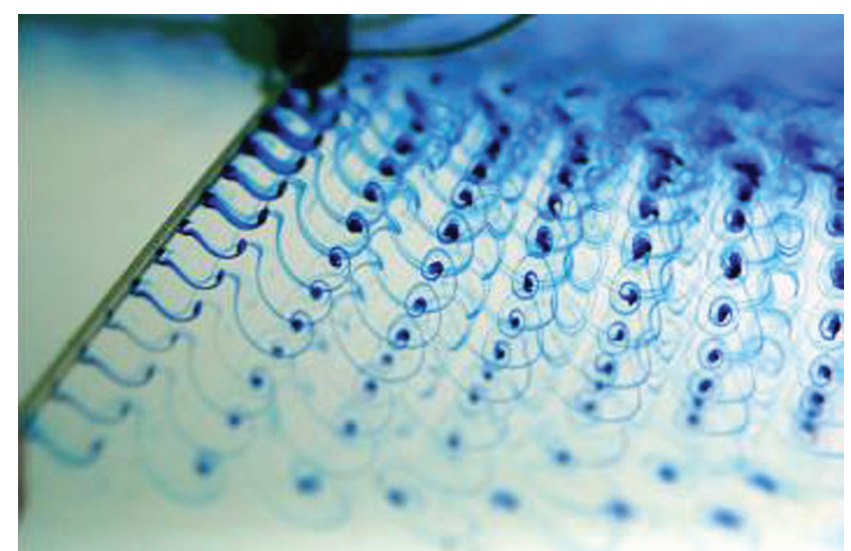

Fig. 16 Flow visualization of the cylinder wake at $R e=120$, forced at the natural shedding frequency with an amplitude of 30 per cent of the cylinder diameter $[\mathbf{1 2 - 1 4}]$
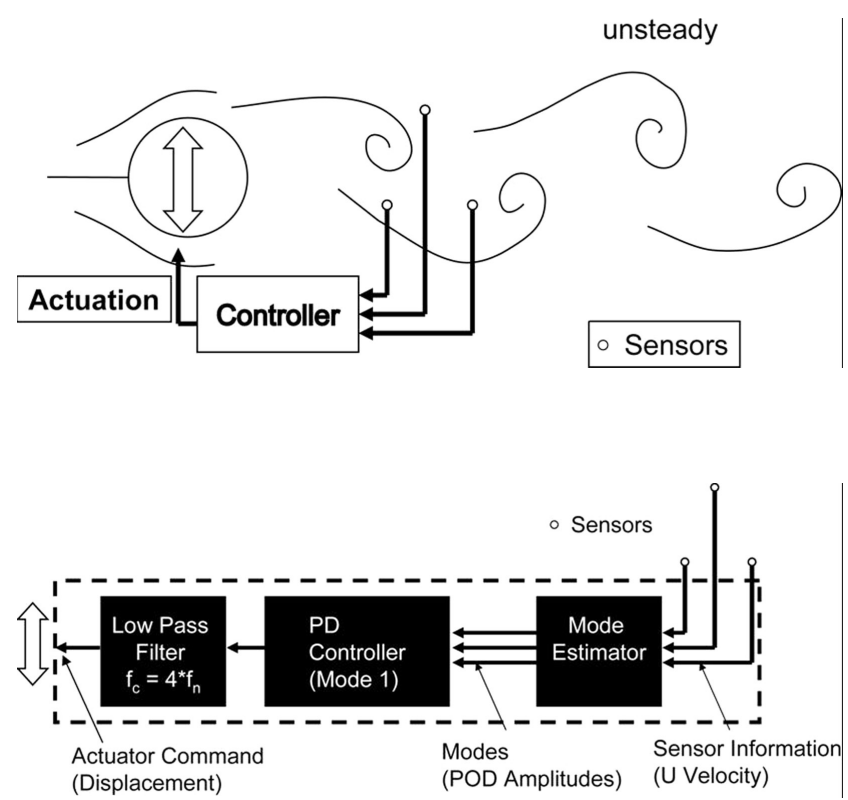

Fig. 17 Flow geometry around a circular cylinder including sensor placement and control concept [12-14]

computed using the Navier-Stokes solver Cobalt, and the POD analysis was done with MatLab. Figure 17 shows the feedback loop after information from MatLab, which is used to oscillate the cylinder normal to the free stream flow to excite or dissipate the vortex wake. The CFD was used to determine optimal number and location of the sensors to accurately (to required levels) describe the flow.

In the unforced flow, the vortices roll up between 1 and 2 diameters downstream of the cylinder, whereas in the feedback-controlled situation, the rollup occurs between 3 and 4 diameters 

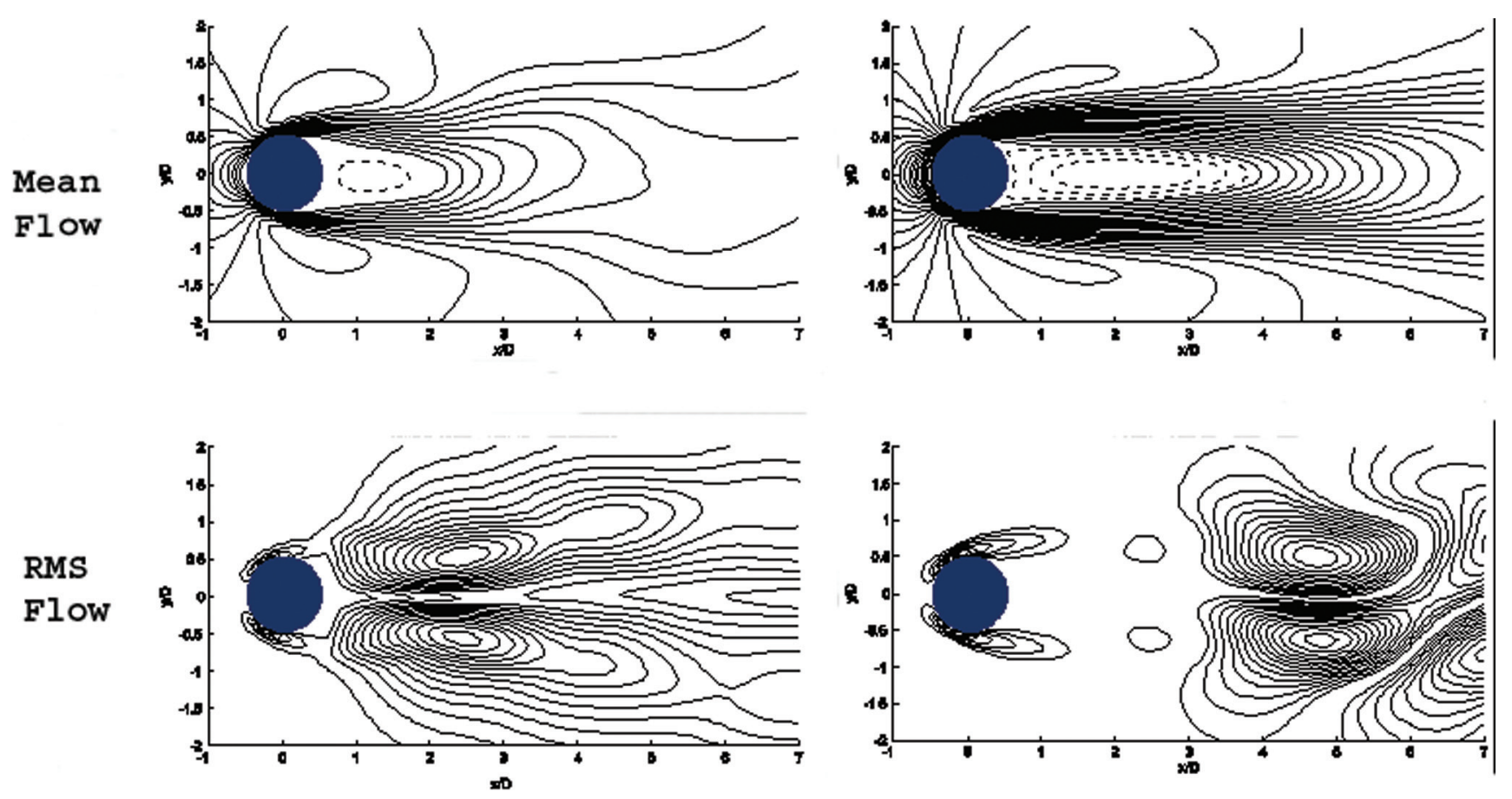

Uncontrolled

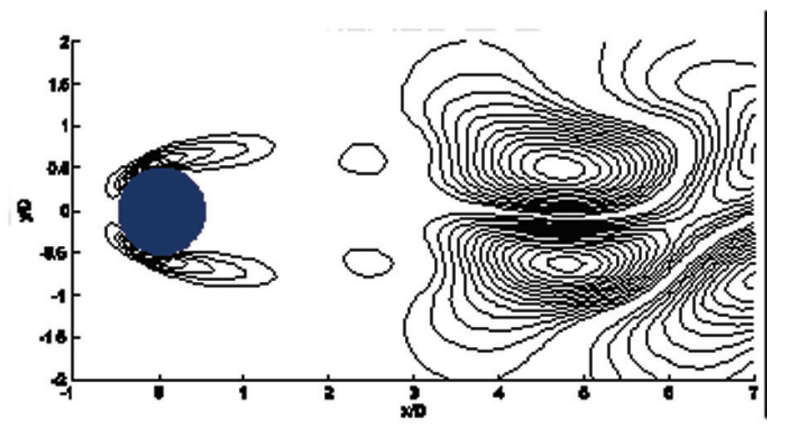

Controlled

Fig. 18 Mean flow (top) and r.m.s. velocity distributions (bottom). Left: uncontrolled case and right: controlled case. The cylinder is centred at $(0,0)$ and of diameter 1 , flow from left to right. Negative isocontours are shown by dashed lines and positive isocontours by solid lines

downstream, as shown in Fig. 18. Simultaneous with the lengthening of the recirculation zone, the researchers observed a reduction in the vortex-shedding frequency. In the low drag state, the near wake is entirely steady, whereas the far wake exhibits vortex shedding at a reduced intensity. The forced case achieved a drag reduction close to 90 per cent of the vortex-induced drag and lowered the unsteady lift force by the same amount.

The success of the low-dimensional feedback control of the circular cylinder wake in the two-dimensional CFD simulation led to the implementation of the control approach in a water tunnel experiment. Reynolds number and actuation of the experiment match the simulation exactly. An in-house-developed real-time PIV system was used to provide sensor information at the same downstream and flow normal locations used in the CFD simulation, using a grid of 35 off-body sensors. The main difference is that the simulation was two dimensional, whereas the water tunnel model features a three-dimensional model and flow field with an aspect ratio of more than 40 . The simulation setup shown in Fig. 19 resembles the water tunnel experiment in terms of aspect ratio, Reynolds number, and feedback control method employed. With these experimental findings, three-dimensional numerical

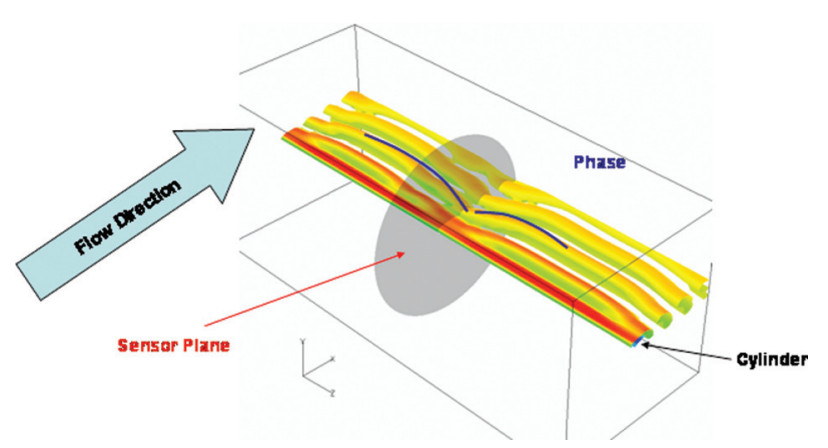

Fig. 19 Numerical simulation setup for threedimensional case [12-14]

simulations were performed to gather quantitative data along the span of the model, which is not possible with the current state-of-the-art experimental measurement techniques. In this way, the CFD will provide information about the essential twodimensional features of the flow, while still exhibiting the important three-dimensional variations.

In this way, the experiments and computations are being used to inform and improve each other (first experiment, then numerically simulate, followed by improved experiment, etc.). Not only did this approach allow the researchers to test out their 
control concepts in a computational simulation prior to beginning experimentation, but also the CFD provided the detailed placement of sensors and creation of the control system. The experiment was not just used to validate the CFD, it was then used to extend the computational results and start the 'feedback' loop again, with information gained from one perspective being used to improve the other. This approach shows the highest level of integration of CFD and experiments that the authors have seen and shows what can be accomplished when the best attributes of each approach are used together.

\section{CONCLUSIONS}

Five cases of $\mathrm{CFD} /$ experimental interaction have been presented, with each case showing a different way in which research is improved by collaboration. Although the early examples merely show companion studies of CFD and wind tunnel tests, even in this simplest form of collaboration, a great deal is to be gained in understanding. As the collaboration has improved, the interaction has continuously evolved and matured, leading to new ways to interact and improve aerodynamic concept development. Unfortunately, most research is only performed from an experimental or a computational viewpoint, leaving us to wonder how much improvement in aerodynamic concept development collaboration could bring about.

Here are just some examples of what can be done with collaboration (these are also partially based on the 2003 Glasgow symposium findings):

(a) knowledge of the flow before deciding what should be measured;

(b) knowledge of model geometry accuracy and fidelity;

(c) having checks in place on the experimental measurements as they are taken;

(d) overcoming difficulty in making certain important measurements;

(e) assessment of the influence of the experimental techniques on the measurements;

(f) the ability of CFD to provide detailed flow information and sensitivity at a reasonable cost for some cases;

(g) CFD validation and improvement enhanced by good experiments.

Unfortunately, there are still a number of problems that exist when trying to collaborate:

(a) the large cost (and time) of CFD calculations for certain cases (especially massively separated flows); (b) the lack of credibility for CFD results for some flow categories (transition, shock/boundary layer interactions, chemically reacting flows, etc.);

(c) the large cost (and time) in conducting certain experiments.

The authors believe that collaboration is only limited by our imagination and level of determination. If the experiments and CFD can be visualized as being complimentary, they can be used to bring out the best attributes of each. In fact, the strengths and weaknesses of the two approaches are significantly inter-linked, so good research should often require a collaborative approach.

\section{ACKNOWLEDGMENTS}

The authors wish to thank Stefan Siegel of the USAF Academy for the flow control simulation information and Bruno Vignes and Yannick Bury of the ENSICA for providing the C-130 information. We also thank Boeing Military Aircraft in St Louis, MO, USA for providing the UCAV 1301 geometry.

\section{REFERENCES}

1 Chapman, D. R. Aerodynamic analysis requiring advanced computers. NASA SP-347, 1975, pp. 4-7.

2 Cummings, R. M., Morton, S. A., and Siegel, S. G. Computational simulation and PIV measurements of the laminar vortical flowfield for a delta wing at high angle of attack. AIAA 41st Aerospace Sciences Meeting, January 2003, AIAA paper 2003-1102 (AIAA, Reston, VA).

3 Cummings, R. M., Morton, S. A., and Siegel, S. G. Computational simulation and experimental measurements for a delta wing with periodic suction and blowing. $J$. Airc., 2003, 40(5), 923-931.

4 Strang, W. Z., Tomaro, R. F., and Grismer, M. J. The defining methods of Cobalt $_{60}$ : a parallel, implicit, unstructured Euler/Navier-Stokes flow solver. AIAA 37th Aerospace Sciences Meeting, January 1999, AIAA paper 99-0786 (AIAA, Reston, VA).

5 Tomaro, R. F., Strang, W. Z., and Sankar, L. N. An implicit algorithm for solving time dependent flows on unstructured grids. AIAA 35th Aerospace Sciences Meeting, January 1997, AIAA paper 97-0333 (AIAA, Reston, VA).

6 Grismer, M. J., Strang, W. Z., Tomaro, R. F., and Witzemman, F. C. Cobalt: a parallel, implicit, unstructured Euler/Navier-Stokes solver. Adv. Eng. Softw., 1998, 29(3-6), 365-373.

7 Forsythe, J. R., Strang, W. Z., and Hoffmann, K. A. Validation of several Reynolds-averaged turbulence models in a 3D unstructured grid code. AIAA Fluids 2000 Conference, June 2000, AIAA paper 2000-2552 (AIAA, Reston, VA). 
8 Morton, S. A. High Reynolds number DES simulations of vortex breakdown over a 70 degree delta wing. AIAA 21st Applied Aerodynamics Conference, June 2003, AIAA paper 2003-4217 (AIAA, Reston, VA).

9 Cummings, R. M., Morton, S. A., Siegel, S. G., and Bosscher, S. Numerical prediction and wind tunnel experiment for a pitching unmanned combat air vehicle. AIAA 41st Aerospace Sciences Meeting, January 2003, AIAA paper 2003-417 (AIAA, Reston, VA).

10 Florendo, C., Yechout, T., Siegel S. G., Cummings, R. M., and Kealos, J. Experimental evaluation of a high fineness ratio body with drag brakes. AIAA 44th Aerospace Sciences Meeting, January 2006, AIAA paper 2006-0666 (AIAA, Reston, VA).

11 Cummings, R. M., Divine, J. A., Yechout, T., Wetlesen, D., and Kealos, J. Numerical evaluation of the flowfield for a high fineness ratio body with drag brakes. AIAA 44th Aerospace Sciences Meeting, January 2006, AIAA paper 2006-0668 (AIAA, Reston, VA).

12 Siegel, S., Cohen, K., and McLaughlin, T. Feedback control of a circular cylinder wake in experiment and simulation. AIAA 33rd Fluid Dynamics Conference, June 2003, AIAA paper 2003-3569 (AIAA, Reston, VA).

13 Siegel, S., Cohen, K., and McLaughlin, T. Experimental variable gain feedback control of a circular cylinder wake. AIAA 24th Aerodynamic Measurement Technology and Ground Testing Conference, June 2004, AIAA paper 2004-2611 (AIAA, Reston, VA).

14 Seidel, J., Siegel, S., Cohen, K., and McLaughlin, T. Three dimensional simulations of a feedback controlled circular Cylinder Wake. AIAA 43rd Aerospace Sciences Meeting, January 2005, AIAA paper 2005-0295 (AIAA, Reston, VA). 